\title{
Physical Activity and Daily Nutrient Intake for Prevention of Lifestyle-Related Diseases in Individuals with Spinal Cord Injury
}

\author{
Hiroshi Ohko*1, Fumie Kajihara ${ }^{2}$, Takahiro Hayashi ${ }^{1}$, Akira Ochi $^{1}$, Keiko Kumagawa ${ }^{3}$ and Hiroyuki \\ Okawa $^{4}$ \\ ${ }^{1}$ Department of Rehabilitation and Care, Seijoh University, Japan \\ ${ }^{2}$ Osaka General Medical Center, Japan
}

${ }^{3}$ Department of Health and Nutrition Sciences, Faculty of Health and Nutrition Sciences, Nishikyushu University, Japan

${ }^{4}$ Department of Rehabilitation, Nishikyushu University, Japan

*Corresponding author: Hiroshi Ohko, Department of Rehabilitation and Care, Seijoh University, 2-172 Fukinodai, Tokai, Aichi, 476-8588, Japan

\section{ARTICLE INFO}

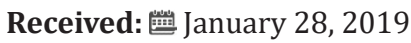

Published: 幽 February 05, 2019

Citation: Ohko H, Kajihara F, Hayashi T, Ochi A, Kumagawa K, Okawa H. Physical Activity and Daily Nutrient Intake for Prevention of Lifestyle-Related Diseases in Individuals with Spinal Cord Injury. Biomed J Sci \& Tech Res 14(1)-2019. BJSTR. MS.ID.002495.

Keywords: Spinal Cord Injury; Physical Activity; Nutrients

\section{ABSTRACT}

Objectives: We aimed to determine the nutritional status of participants with spinal cord injuries through measuring physical activity (heart rate, acceleration) during sport (a wheelchair half marathon) and daily life activities, and we collated data for use in the prevention of lifestyle-related diseases in individuals with cervical spinal cord injury (CSCI).

Study Design and Setting: This cross-sectional study involved selected individuals participating in the Oita International Wheelchair Marathon.

Methods: Eight individuals with CSCI participating in a wheelchair half marathon were included in this study. Physical activity was assessed through measuring heart rate and acceleration using a portable accelerometer during the half marathon and daily heart rate and acceleration were measured again at a later date. We conducted a nutrient intake survey (calories, protein, lipids, vitamin C and iron levels) during a 3-day measurement of daily activities, and intake of all foods including snacks was recorded.

Results: Heart rate and acceleration during the wheelchair half marathon were significantly higher than measures taken during activities of daily living. Protein deficiency $(71.6 \pm 15.3 \%)$ and hyperlipidemia $(121.6 \pm 19.6 \%)$ were observed in all participants, while deficiencies of vitamin $\mathrm{C}$ and iron were observed in some participants. Dietary intake in some participants was limited because of elimination disorders.

Conclusion: This study examined heart rate and acceleration during sporting and daily life activities in relation to nutrient intake in individuals with CSCI and provided basic information useful for the prevention of lifestyle-related diseases. Further research is needed to prevent lifestyle-related disease and improve competitiveness.

\section{Introduction}

In recent years, tailored training and nutrition management programs have been implemented for top-level athletes with physical disabilities, such as those competing in the Paralympic
Games. The application of training support programs has also been disseminated among non-athletes with physical disabilities. 
Spinal cord injury (SCI) causes a decrease in muscle mass due to paralysis arising from nerve damage, and also leads to a relative increase in body fat mass. In addition, a reduction in physical activity causes glucose/lipid metabolism disorders, which are related to the onset of cardiovascular events [1-2]. In general, as individuals with SCI age, they are at an increased risk of lifestyle-related diseases [3-4]. Moreover, the basal metabolic rate in individuals with SCI in the chronic phase is reportedly $21 \%$ lower than in healthy individuals [5]. It has been assumed that individuals with SCI have a higher risk of increased body fat and lifestyle-related diseases if their eating habits are similar to those of healthy individuals, due to the differences in energy intake and expenditure between the two groups [6]. In individuals with cervical SCI (CSCI) and decreased muscle mass due to paralysis exercise content is particularly limited and risks may be even higher. Thus, individuals with CSCI are also more in need of comprehensive support for sports activities and dietary intake than those with other SCIs. However, few studies have been conducted concerning daily nutritional intake in nonathlete CSCI patients.

Thus, we collected basic data for use in the prevention of lifestyle-related diseases in individuals with CSCI. We measured physical activity (heart rate, acceleration) in one sporting activity (a wheelchair half marathon) and in daily life. Furthermore, we conducted a survey of daily nutrient intake and determined the nutritional status of the study participants.

\section{Methods}

Eight males with CSCI who participated in the $35^{\text {th }}$ or $36^{\text {th }}$ Oita International Wheelchair Marathon were included in this study. The participant characteristics are shown in Table 1. Physical activity was assessed through measurement of heart rate and acceleration during competition, using a portable accelerometer (ACTIVETRACER AC301; GMS Co., Tokyo, Japan) [7] and data were collected during the wheelchair half marathon. Calorie consumption and metabolic equivalents (METs) during the half marathon were calculated from acceleration values [8].

Table 1: Participant characteristics.

\begin{tabular}{|c|c|}
\hline & Wheelchair half marathon $(\mathbf{n}=\mathbf{8})$ \\
\hline Age $(\mathrm{yr})$ & $36.8 \pm 7.0$ \\
\hline Height $(\mathrm{cm})$ & $171.4 \pm 5.8$ \\
\hline Weight $(\mathrm{kg})$ & $57.2 \pm 13.6$ \\
\hline BMI $\left(\mathrm{kg} / \mathrm{m}^{2}\right)$ & $19.4 \pm 3.8$ \\
\hline SCI Level $/$ Class & $\mathrm{C} 5,1 ; \mathrm{C} 6,6 ; \mathrm{C} 7,1$ \\
\hline Disease duration $(\mathrm{yr})$ & $11.7 \pm 8.5$ \\
\hline
\end{tabular}

Values are mean \pm standard deviation.

BMI, body mass index; SCI, spinal cord injury

A belt was used to attach a portable accelerometer to the center of the abdomen beneath the navel. To determine heart rate, bipolar chest leads were used to measure R-R intervals, which were then averaged over one minute. Physical activity of $\geq 0.05-G$ acceleration was recorded every minute with synthesized acceleration in three directions (i.e., $x, y$, and z). Daily mean heart rate and acceleration data were calculated and used for analysis. During the measurement of daily activities, we also surveyed sports participation.

The survey of nutrient intake was performed during a 3-day measurement of daily activities, and the intake of all foods including snacks was recorded. Measurements were undertaken between 06:00h and 21:00h for 3 days. To record intake, a dish was photographed before and after meals alongside a ruler, from a 60-degree angle, to document its depth. Each dish was also photographed prior to the intake of main and side dishes. We also surveyed the intake of seasonings and water, and cooking methods, to estimate nutrient intake. Recommended daily energy intake was calculated through multiplying the basal metabolic rate by the daily physical activity intensity (physical activity $\times 1.4$ ) [9]. Basal metabolic rate was defined as $80 \%$ of the product of the basal metabolic standardized value for age and sex, as cited in the Japanese Dietary Intake Standards, and standardized body weight [10-12]. Recommended protein intake was defined as that required for endurance athletes (i.e., $1.2-1.4 \mathrm{~g} / \mathrm{kg} /$ day). Recommended lipid intake was defined as the amount recommended for the prevention of lifestyle-related diseases. Recommended vitamin C and iron intake were defined as the amount recommended for healthy individuals. Actual consumption was calculated as a percentage of the recommended nutrient intake.

We performed a statistical analysis of heart rate and acceleration during the wheelchair half marathon, and compared physical activity during the race, physical activity undertaken during daily activities on days with exercise, and physical activity during daily activities on days without exercise, using the Friedman test. The Bonferroni post-hoc test was used, with a significance level of $\mathrm{p}<0.05$. SPSS ver. 17.0 (SPSS, Inc., Chicago, IL, USA) was used to analyze the data. This study complied with the Declaration of Helsinki and was implemented with the approval of the Research Ethics Committee of Seijoh University. The participants were provided with sufficient explanation concerning the study, and written informed consent was obtained prior to performing the study procedures.

\section{Results}

Table 2 shows the results of the wheelchair half marathon. Five of 8 participants completed the half marathon. Based on accelerometer readings, the average energy expenditure during the half marathon was $234.8 \pm 109.0 \mathrm{kcal}$ for participants who completed the half marathon and $78.0 \pm 29.1 \mathrm{kcal}$ for those who withdrew early. Participants' heart rates were highest during the wheelchair half marathon and were significantly higher on days with exercise ( $p<0.01,95 \%$ confidence interval (CI) 0.85-2.50, effect size (ES) 0.86) (Figure 1). Acceleration showed the highest 
value during the wheelchair half marathon and was significantly higher on days with exercise ( $p<0.01,95 \%$ CI $0.44-1.35$, ES 0.75) (Figure 2). Acceleration rates measured during the half marathon, and on days with and without exercise, were $162.7 \pm 46.7 \mathrm{mG} /$ count, $33.7 \pm 4.3 \mathrm{mG} /$ count and $22.1 \pm 6.1 \mathrm{mG} /$ count, respectively. Heart rate measures undertaken during the half marathon and on days with and without exercise, were $107.6 \pm 9.2$ beats per minute (bpm), $78.9 \pm 9.4 \mathrm{bpm}$, and $68.7 \pm 3.5 \mathrm{bpm}$, respectively. Sports activities on days with exercise mainly involved activities such as wheelchair sprinting, wheelchair racing, wheelchair basketball and wheelchair rugby training. The mean duration of the training sessions was approximately between 1 and 2 hours.

Table 2: Results for participants completing the wheelchair half marathon.

\begin{tabular}{|c|c|c|c|c|}
\hline & Participant & Race duration & Ranking (class) & Calorie consumption \\
\hline \multirow{4}{*}{ Half marathon completed } & $\mathrm{A}$ & $1: 17$ & 6 (T52) & 194 \\
\cline { 2 - 5 } & $\mathrm{B}$ & $1: 27$ & $12(\mathrm{~T} 52)$ & 424 \\
\cline { 2 - 5 } & $\mathrm{C}$ & $1: 38$ & $2(\mathrm{~T} 51)$ & 156 \\
\cline { 2 - 5 } & $\mathrm{D}$ & $1: 03$ & $2(\mathrm{~T} 52)$ & 173 \\
\hline \multirow{3}{*}{ Retired early } & $\mathrm{E}$ & $1: 16$ & $9(\mathrm{~T} 52)$ & 227 \\
\cline { 2 - 5 } & $\mathrm{F}$ & $(0: 40)$ & $-(\mathrm{T} 52)$ & 708 \\
\cline { 2 - 5 } & $\mathrm{G}$ & $(0: 30)$ & $-(\mathrm{T} 51)$ & 76 \\
\hline
\end{tabular}

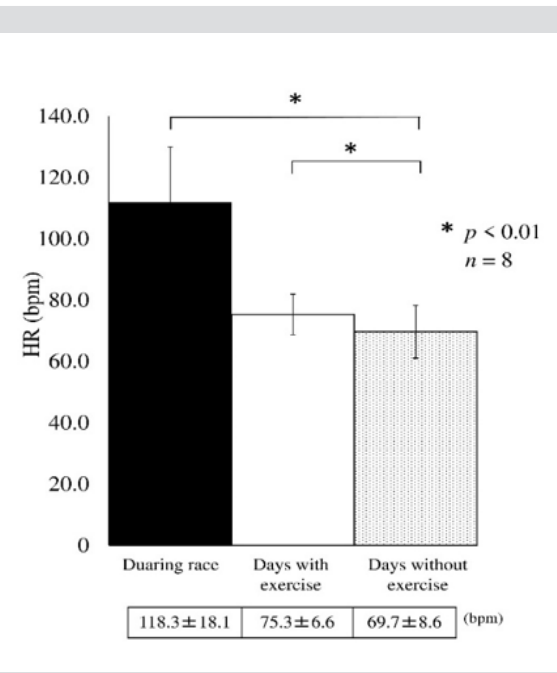

Figure 1: Mean heart rates for the wheelchair half marathon participants.

Values are mean \pm standard deviation.

*Significant difference $\left({ }^{*} p<0.01\right)$ between measurements recorded during the half marathon and on the days with exercise.

Table 3 shows the actual nutrient intake as a percentage of the recommended daily nutrient intake for each participant. Although the participants met the recommended daily energy intake (average, $96.9 \pm 15.1 \%$ ), lipid intake was in excess of the Table 3: Nutrient intake according to each participant (\%).

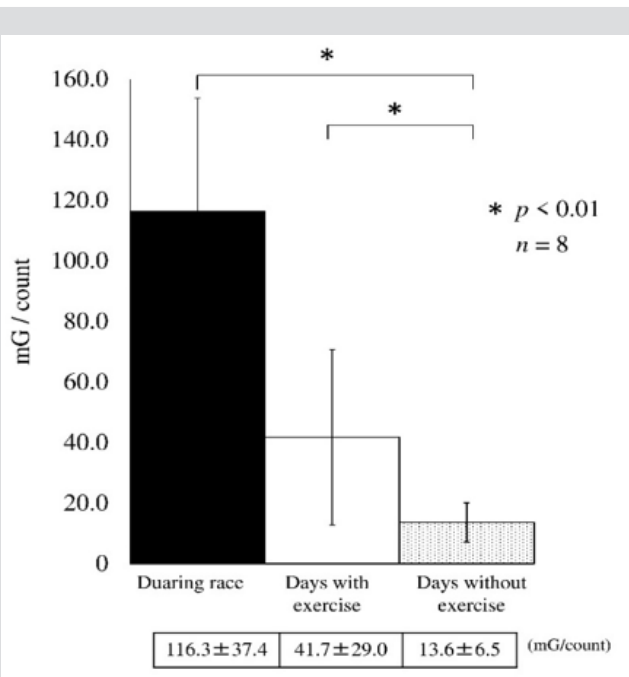

Figure 2: Mean acceleration during the wheelchair half marathon.

Values are mean \pm standard deviation.

*Significant difference $\left({ }^{*} p<0.01\right)$ between measurements recorded during the half marathon and on the days with exercise.

recommended average $(129.0 \% \pm 9.0 \%)$. However, protein intake (average, $74.7 \% \pm 12.9 \%$ ), vitamin $\mathrm{C}$ intake (average, $85.1 \% \pm$ $30.1 \%$ ), and iron intake (average, $86.9 \% \pm 20.2 \%$ ) were lower than the recommended intake for some participants.

\begin{tabular}{|c|c|c|c|c|c|}
\hline No & Energy & Protein & Lipids & Vitamin C & 130.0 \\
\hline A & 103.6 & 96.3 & 137.6 & 49.0 & 118.7 \\
\hline B & 107.5 & 64.9 & 120.4 & 43.0 & 77.3 \\
\hline C & 86.2 & 57.5 & 117.6 & 67.0 & 78.6 \\
\hline D & 81.4 & 66.2 & 144.0 & 105.0 & 65.7 \\
\hline E & 88.5 & 68.4 & 124.8 & 105.0 & 81.4 \\
\hline F & 126.6 & 85.2 & 130.0 & & 100.0 \\
\hline
\end{tabular}




\begin{tabular}{|c|c|c|c|c|c|}
\hline G & 85.3 & 75.5 & 123.6 & 85.0 & 94.0 \\
\hline H & 96.0 & 83.4 & 133.6 & 85.1 & 109.3 \\
\hline AVE & 96.9 & 74.7 & 129.0 & 30.1 & 86.9 \\
\hline SD & 15.1 & 12.9 & 9.0 & 20.2 \\
\hline
\end{tabular}

AVE, average; SD, standard deviation.

\section{Discussion}

This is the first study of daily nutrient intake and physical activity in an Asian population with CSCI, and our results indicated that heart rate and acceleration were significantly higher on days with exercise than on days without exercise.

In a study involving elite Asian Grade A athletes with complete traumatic T4 SCIs, mean heart rate during a wheelchair marathon race was reported to be $137 \mathrm{bpm}$ and the mean calorie expenditure per hour was reported to be $463 \mathrm{kcal} / \mathrm{h}$. The mean heart rate during the wheelchair half marathon in the participants involved in this study was $118.3 \mathrm{bpm}$ and the mean calorie consumption was $234.8 \mathrm{kcal}$ [13]. These results appear to be within the expected range with respect to CSCI patients. Regular exercise through sports activities can increase heart rate, physical activity and energy expenditure in individuals with CSCI.

According to the International SCI Exercise Guidelines Project Group, medium-intensity aerobic exercise should be performed for at least 30 minutes per week in order to maintain cardiovascular function [14]. One epidemiological study found that regular exercise, including sporting events and activities, prevented lifestyle-related diseases and improved abnormal glucose and lipid metabolism, even in individuals with CSCI [15]. As competitions and training activities increase day-to-day physical activity, such activity may prevent the onset of lifestyle-related diseases.

In a survey of workers with SCIs, regular participation in leisure sports was found to have increased the workers' maximum oxygen intake. Regular exercise may increase not only energy consumption but also maximum oxygen intake [16]. However, because the maximum oxygen intake in individuals with CSCI is lower than in those with other SCIs, routine daily activities may not provide sufficient physical activity. In individuals with CSCI in particular, there is a tendency to spend large amounts of time on everyday activities and, as such, there is a need to promote increased physical activity and engagement in sporting activities after implementing lifestyle management measures to reduce self-care activities [17]. As such, sports activities may be even more important for those with CSCI.

This study determined the actual status of nutrient intake in individuals with CSCI. Although actual energy intake met the recommended daily requirements, some participants had hyperlipidemia, along with protein, vitamin C, and iron deficiencies, and a diet associated with lifestyle-related diseases. We found that some participants skipped meals to reduce the frequency of excretion. Thus, elimination disorders may influence dietary intake. A reduction in nutritional intake leading to the onset of gastrointestinal issues has been also been reported concerning individuals with CSCI [18]. These results suggest that increasing physical activity through daily sports participation and managing nutrient intake, with an emphasis on a balanced diet, may be necessary for the prevention of lifestyle-related diseases in individuals with CSCI.

This study had some limitations. First, the daily nutrient requirements were based on data derived from healthy participants in a previous report by Cooper et al. [5]. Moreover, guidelines for nutritional management in acute CSCI have stated that the values from analysis of exhaled gas are preferable to those calculated from height and body weight [19]. In addition, an analysis of metabolism at rest in individuals with SCIs using a simple calorimeter showed that recommended daily energy intake calculated using the HarrisBenedict formula or "Dietary Reference Intakes for Japanese" was between $300 \mathrm{kcal}$ and $500 \mathrm{kcal}$ more than the actual measured values [20]. Thus, there may be large individual differences in recommended energy intake due to differences in muscle mass and physical activity [21], depending on the level of spinal injury [22].

To accurately calculate recommended nutrient intake for individuals with CSCI, an interventional study of metabolic rate, energy intake, various nutrients, and eating habits using direct measurement methods, such as analysis of exhaled gas, is required in the future. The results of this study suggest that physical activity, training, and nutritional support that takes into account eating habits is necessary and beneficial for individuals with CSCI, including those who may participate, for example, in wheelchair half marathons.

\section{Acknowledgement}

We would like to express our sincere gratitude to the participants of the Oita International Wheelchair Marathon and the members of Fukuoka Dandelion who contributed to this study. This work was supported by JSPS KAKENHI Grant Number JP26350798, JP17K13077.

\section{References}

1. Janssen TW, van Oers CA, Veeger HE, Hollander AP, van der Woude LH, et al. (1994) Relationship between physical strain during standardized ADL tasks and physical capacity in men with spinal cord injuries. Paraplegia 32(12): 844-859. 
2. Schönherr MC, Groothoff JW, Mulder GA, Eisma WH (2005) Participation and satisfaction after spinal cord injury: Results of a vocational and leisure outcome study. Spinal Cord 43(4): 241-248.

3. de Groot S, Adriaansen JJ, Tepper M, Snoek GJ, van der Woude LH, et al. (2016) Metabolic syndrome in people with a long-standing spinal cord injury: Associations with physical activity and capacity. Appl Physiol Nutr Metab 41(11): 1190-1996.

4. Groah SL, Weitzenkamp D, Sett P, Soni B, Savic G (2001) The relationship between neurological level of injury and symptomatic cardiovascular disease risk in the aging spinal injured. Spinal Cord 39(6): 310-317.

5. Cooper IS, Rynearson EH, MacCarty CS, Power MH (1950) Metabolic consequences of spinal cord injury. J Clin Endocrinol Metab 10(8): 858870.

6. Yokose Y, Tsunoda N, Inayama T, Fumiko S, Jun O, et al. (2007) Body characteristics and dietary life in persons with spinal cord injury. The $39^{\text {th }}$ Conference of the Asia-Pacific Academic Consortium for Public Health, Japan, pp. 179.

7. Kawahara J, Tanaka S, Tanaka C, Aoki Y, Yonemoto J (2011) Estimation of daily inhalation rate in preschool children using a tri-axial accelerometer: A pilot study. Sci Total Environ 409(16): 3073-3077.

8. Hikihara Y, Tanaka S, Ohkawara K, Ishikawa-Takata K, Tabata I (2012) Validation and comparison of 3 accelerometers for measuring physical activity intensity during non-locomotive activities and locomotive movements. J Phys Act Health 9(7): 935-943.

9. Krempien JL, Barr SI (2011) Risk of nutrient inadequacies in elite Canadian athletes with spinal cord injury. Int J Sport Nutr Exerc Metab 21(5): 417-425.

10. Ganpule AA, Tanaka S, Ishikawa-Takata K, Tabata I (2007) Interindividual variability in sleeping metabolic rate in Japanese subjects. Eur J Clin Nutr 61(11): 1256-1261.

11. Ministry of Health, Labour and Welfare (2015) Overview of Dietary Reference Intakes for Japanese.

\section{ISSN: 2574-1241}

DOI: 10.26717.BJSTR.2019.14.002495

Hiroshi Ohko.Biomed J Sci \& Tech Res

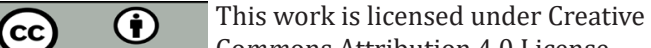
Commons Attribution 4.0 License

Submission Link: https://biomedres.us/submit-manuscript.php
12. Levine AM, Nash MS, Green BA, Shea JD, Aronica MJ (1992) An examination of dietary intakes and nutritional status of chronic healthy spinal cord injured individuals. Paraplegia 30(12): 880-889.

13. Abel T, Schneider S, Platen P, Strüder HK (2006) Performance diagnostics in handbiking during competition. Spinal Cord 44(4): 211-216.

14. Goosey-Tolfrey VL, Scheer JW, Lexell J, Clements K, Ginis KA (2018) Development of scientific exercise guidelines for adults with spinal cord injury. Br J Sports Med 52(18): 1166-1167.

15. Hoffman MD (1986) Cardiorespiratory fitness and training in quadriplegics and paraplegics. Sports Med 3(5): 312-330.

16. Tajima F, Ogata H, Mizushima T, Nakamura T, Nagano A (2001) Agerelated medical issues in workers with physical disabilities. Backhuys Publishers, Leiden, Netherlands, pp. 201-212.

17. Perret C, Stoffel-Kurt N (2011) Comparison of nutritional intake between individuals with acute and chronic spinal cord injury. J Spinal Cord Med 34(6): 569-575

18. Kato M, Tajika M, Miwa Y, Moriwaki H (2002) Validation of a portable indirect calorimeter (Metavine) for measuring energy expenditure in an elderly population. Clin Exp Pharmacol Physiol Suppl 29: S9-S12.

19. Takayo I, Yukie Y, Nobuyo T, Hisako U, Hajimu S, et al. (2013) Measurement of resting energy expenditure and dietary planning for persons with spinal cord injury. Jpn J Nutr Diet 51: 59-66.

20. Oettlé GJ (1991) Effect of moderate exercise on bowel habit. Gut 32(8): 941-944.

21. Alexander LR, Spungen AM, Liu MH, Losada M, Bauman WA (1995) Resting metabolic rate in subjects with paraplegia: The effect of pressure sores. Arch Phys Med Rehabil 76(9): 819-822.

22. Cox SA, Weiss SM, Posuniak EA, Worthington P, Prioleau M, et al. (1985) Energy expenditure after spinal cord injury: An evaluation of stable rehabilitating patients. J Trauma 25(5): 419-423.

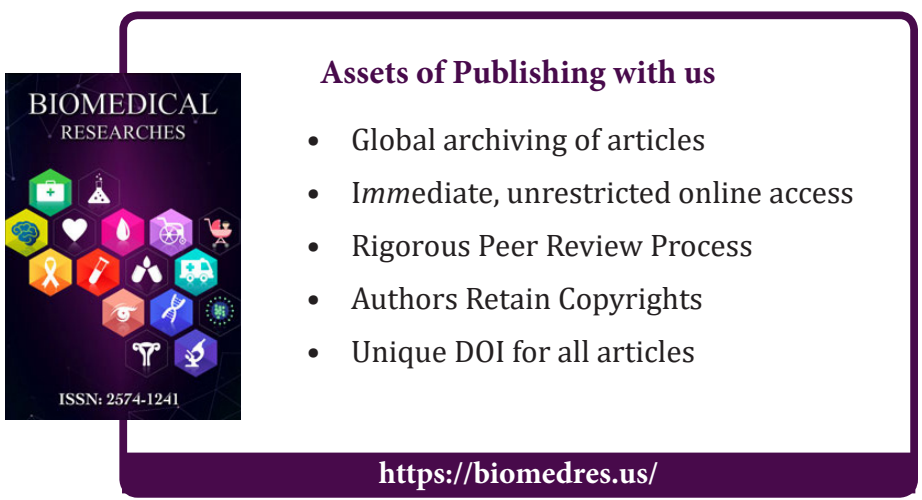

